\title{
Upgrading from One-way Informing to Two-way Audience- oriented Health Communication: CFSA Initiations for World Food Safety Day
}

\author{
Si Chen'; Juana $\mathrm{Du}^{2}$; Fangmin Gong³ ; Hongwei Han'; Jianwen $\mathrm{Li}^{1}$; \\ Jinjun Liang ${ }^{4}$; Patrick Wall'; Yongning $\mathrm{Wu}^{1, *}$; Zhenyi $\mathrm{Li}^{2, *}$
}

Food safety is vital to the development of human society. On December 20, 2018, the United Nations General Assembly adopted resolution 73/250 proclaiming a World Food Safety Day. As of 2019, every June 7 is a time to increase social awareness of food safety and to encourage actions for good health promotion. The Third World Food Safety Day on June 7, 2021 aims to draw attention and inspire action to help prevent, detect, and manage foodborne risks, contributing to food security, human health, economic prosperity, agriculture, market access, tourism, and sustainable development ( $(1)$.

Food safety risk communication shifts from the traditional approach of one-way sender-oriented to a two-way audience-oriented communication approach. International organizations have achieved consensus that recent advancement of technologies and institutions fundamentally impact how the public perceive, communicate, and react to food safety risk issues. It is crucial to conduct audience analysis to gain a comprehensive understanding of risk perception and communication. For instance, the European Food Safety Authority (EFSA) recommended an audience analysis approach for food-related risk communication practices. Data-driven insights are encouraged by EFSA's Social Science Roadmap (2019-2021) (2). It is key to identify and segment audiences, to measure understanding of public information, and to tailor communication methods. The importance of producing and delivering public information through partnership approach and social media integration are also emphasized (3).

\section{FOOD SAFETY RESEARCH AND PRACTICE FROM CFSA}

China National Center for Food Safety Risk Assessment (CFSA) has been conducting research and directing practice on risk communication, including food-borne disease and mortality caused by poisonous mushrooms and microorganisms because they are reported as high lethality in certain regions in China (4). For example, poisonous mushroom adds enormous burden to local health systems and causes economic loss to the families and communities (5). Common public health campaign attempting to dissuade mushroom picking and eating has not made very effectively changes as the cases of illness and mortality have remained high in many regions for years (G).

Seeing the absence of systematic examination on risk communication, the research team initiated an exploration on this issue by collecting data from the field as well as reviewing relevant literature for applicable theories since 2020 . The team has collected 1,262 questionnaires from Hunan, Guizhou, Jiangxi, Chongqing, and Yunnan where mushroom poisoning cases are concentrated. The team also conducted indepth interviews in Hunan and Yunnan for supplementary understanding of the quantitative data analysis results. Meanwhile, the team has surveyed mainstream health communication theories for a rational model that could guide our research design, data analyses, and recommendation implementation.

The major findings from empirical data identified the high-risk population as local low-income, loweducation, agricultural-oriented with high-level of optimistic bias who are over-confident of their mastery of knowledge and skills to differentiate non-poisonous mushrooms from the poisonous ones based on their experiences and knowledge exchange with peers ( 6 ). Most of this population intend to assess risks by themselves, and they intend to interpret their own risk in a self-serving manner (7). This explains why common public health campaign could hardly penetrate and reach this population.

The team also found that those who received the health campaign messages were not ready to accept the message or to decide to take actions. This indicates the high-risk population is concentrated on the 
counterproductive side on the 5C-model (8) (confidence, calculation, complacency, constraints, and collective responsibility). The team also found our data and findings fit well with Massive Persuasion for Behavioral Change Model (9), Health Belief Model (10), Protection Motivation Theory (11), Theory of Planned Behavior (12), and Social Cognitive Theory (13). These theories all point out that the high-risk population are at the early stage of behavioral change with great probability to reject health campaign messages.

Food safety is not simply related to food consumption. For example, in our research on why people neglected risks related to poisonous mushroom, we identified 4 stumbling barriers for these population to make behavioral change based on the Health Belief Model: 1) knowledge; 2) belief; 3) habits; and 4) culture. None of these four barriers can be overcome overnight by a law or a motion. All barriers are embedded deeply in local contexts. Therefore, it is important to conduct contextual analysis on how to communicate these risks to the local people before launching any health promotion campaign. A one-forall message can neither penetrate the population nor convince them for behavioral change. In Hunan, for example, we suggested differentiating communication strategies to regions with different risk levels. A map of poisonous mushroom risks becomes the base for selecting these strategies, from general awareness campaign in low-risk zones to specific prevention in high-risk zones. A traffic-light-like signage is now used to send clear visible messages to the public to distinguish up mushroom with high, middle, and lowpoisonous-risk with red, yellow, and green colors, respectively. The team has identified that the top three information sources of high-risk population were TV, WeChat, and short videos. Based on the current findings, we recommend creating accurate messages and using diversified communication channels to target different audience groups. Instead of a traditional oneway communication method adopted by most popular science programs, we encourage creating and distributing interactive messages using Chinese social media channels, such as Kwai, to better engage different audience groups. Following the emotional appeal approach, the team produced several short videos targeting at different age groups, such as A Second Thought For Life targeting at senior groups and a rap music video targeting youth in the high-risk population. These are showcased in the 2021 World Food Safety Day exhibition.
CFSA has been collaborating with several national popular science platforms and TV programs to increase social awareness of food-borne risks such as microorganism. CFSA has designed several interactive games and WeChat mini-apps to meet different social needs, such as "Are You Ready to Cook." Participants only need one minute to play the game from which they learn about the problems of as well as solutions to microorganism risks in food processing. CFSA has developed a popular science interactive game "Secrets Behind Foods" to guide audiences to discover illness caused by microorganisms in familiar circumstances in their daily life. CFSA also produced seven mini scientific videos on foodborne disease related topics to increase public awareness and engagement.

CFSA also assisted to promote products for different audience in 2021 World Food Safety Day including but not limited to: Healthy China Action Plan (2019-2030)" and "the National Nutrition Plan (2017-2030)" for professionals, food-borne disease and food nutrition programs with China Central TV for the public, and popular science picture books and short videos for children.

\section{CHALLENGES AND FUTURE WORK}

The media ecosystem in China has recently experienced dramatic changes due to advances in artificial intelligence (AI), Internet of things (IOT), and $5 \mathrm{G}$ telecommunication. Traditional media yield their influence and power to new media, which can target audiences and tailor content with greater customer stickiness. Food safety messages can take advantage of these changes as segmentation of audiences and tailor-made content become vital for communication appropriateness and efficiency. The key is how communicators respond to these changes. Here are some thoughts for future work:

First, food safety and health communication must be shifted from one-way sender orientation to two-way audience orientation. Health communication is not only for reaching the audience, but also for influencing their behavioral changes. The relationship between communicators and the audience is not superiorsubordinate but mutually beneficial. Therefore, communicators must approach the audience more proactively to understand their risk perceptions before communicators send them messages.

Second, food safety and health communication must be based on valid and reliable scientific knowledge. Communicators must identify how much our audience 
has already grasped, wants to learn more about, and needs to know. Audience interest, trust, and receptivity are all vital for success of health communication.

Third, food safety health communication must respect audience reception rules, where listening, emotional understanding, and empathy all are vital. communicators must understand the audience before designing and delivering messages for better reception and comprehension.

Conflicts of interest: No conflict of interests was reported.

Funding: National Key Research and Development Program of China (Grant number 2017YFC1601502 and 2018YFC1603100).

doi: $10.46234 / \mathrm{ccdcw} 2021.133$

\# Corresponding authors: Yongning Wu, wuyongning@cfsa.net.cn; Zhenyi Li, zhenyi.li@royalroads.ca.

\footnotetext{
${ }^{1}$ National Center for Food Safety Risk Assessment, Beijing, China. National Health Commission Key Laboratory of Food Safety Risk Assessment, Food Safety Research Unit (2019RU014) of Chinese Academy of Medical Science, Beijing, China; ${ }^{2}$ Royal Roads University, Victoria, Canada; ${ }^{3}$ Jishou University, Jishou, Hunan, China; ${ }^{4}$ Hunan Disease Prevention and Control Center, Changsha, China; ${ }^{5}$ University College Dublin, Dublin, Ireland.
}

Submitted: May 25, 2021; Accepted: June 10, 2021

\section{REFERENCES}

1. WHO. World food safety day 2021. https://www.who.int/wester npacific/news/events/detail/2021/06/07/western-pacific-events/worldfood-safety-day-2021\#:- :text=World\%20Food\%20Safety\%20Day
$\% 202021 \% 207 \% 20 \mathrm{June} \% 202021$,prosperity $\% 2 \mathrm{C} \% 20$ agriculture $\% 2 \mathrm{C}$ $\% 20$ market $\% 20$ access $\% 2 \mathrm{C} \% 20$ tourism $\% 20$ and $\% 20$ sustainable $\% 20 \mathrm{de}$ velopment. [2021-5-26].

2. EFSA (European Food Safety Authority). 2019 Eurobarometer on food safety in the EU. 2019. https://www.food-safety.com/articles/64642019-eurobarometer-on-food-safety-in-the-eu. [2021-5-26].

3. Smith A, Vrbos D, Alabiso J, Healy A, Ramsay J, Gallani B. Future directions for risk communications at EFSA. EFSA J 2021;19 (2):e190201. http://dx.doi.org/10.2903/j.efsa.2021.e190201.

4. Sun CY. Information regarding poisonous mushroom. 2020. https:// cfsa.net.cn/Article/News.aspx?id=22F26A3E175424631AF433A1DB55 DBE317D0F69FEE031C64. (In Chinese). [2021-5-26].

5. Alcorn T, Ouyang YD. China's invisible burden of foodborne illness. Lancet 2012;379(9818):789 - 90. http://dx.doi.org/10.1016/S01406736(12)60330-4.

6. Weinstein ND. Unrealistic optimism about susceptibility to health problems: conclusions from a community-wide sample. J Behav Med 1987;10(5):481 - 500. http://dx.doi.org/10.1007/BF00846146.

7. Morton TA, Duck JM. Communication and health beliefs: mass and interpersonal influences on perceptions of risk to self and others. Commun Res 2001;28(5):602 - 26. http://dx.doi.org/10.1177/009365 001028005002 .

8. Betsch C, Schmid P, Heinemeier D, Korn L, Holtmann C, Böhm R. Beyond confidence: development of a measure assessing the $5 \mathrm{C}$ psychological antecedents of vaccination. PLoS One 2018;13 (2):e0208601. http://dx.doi.org/10.1371/journal.pone.0208601.

9. Cartwright D. Risk taking by individuals and groups: an assessment of research employing choice dilemmas. J Pers Soc Psychol 1971;20 (3):361 - 78. http://dx.doi.org/10.1037/h0031912.

10. Rosenstock IM, Strecher VJ, Becker MH. Social learning theory and the health belief model. Health Educ Q 1988;15(2):175 - 83. http://dx. doi.org/10.1177/109019818801500203.

11. Prentice-Dunn S, Rogers RW. Protection motivation theory and preventive health: beyond the health belief model. Health Educ Res 1986;1(3):153 - 61. http://dx.doi.org/10.1093/her/1.3.153.

12. Ajzen I. Perceived behavioral control, self-efficacy, locus of control, and the theory of planned behavior. J Appl Soc Psychol 2002;32(4):665 83. http://dx.doi.org/10.1111/j.1559-1816.2002.tb00236.x.

13. Bandura, A. Health promotion by social cognitive means. Health Educ Behav 2004，31，143-164. http://dx.doi.org/10.1177/1090198104 263660 